\title{
Concurrency benefits in the attentional blink: Attentional flexibility and shifts of decision criteria
}

\author{
Richard Lapointe-Goupil • Claudette Fortin • \\ Benoit Brisson - Sébastien Tremblay
}

Published online: 30 November 2010

(C) Psychonomic Society, Inc. 2010

\begin{abstract}
Fundamental limitations in performing multiple tasks concurrently are well illustrated by the attentional blink $(\mathrm{AB})$ deficit, which refers to the difficulty in reporting a second target (T2) when it is presented shortly after a first target (T1). Surprisingly, recent studies have shown that the $\mathrm{AB}$, which is often thought of as a manifestation of capacity limitations in central processing, can be reduced when the $\mathrm{AB}$ task is performed simultaneously with concurrent distracting activities. In the present study, we sought to investigate whether such concurrency benefits would also be observed when the $\mathrm{AB}$ task was performed concurrently with a central demanding timing task. The $\mathrm{AB}$ was reduced under concurrent-task conditions, as compared with single-AB-task conditions, even though $\mathrm{T} 1$ performance was unaffected by the concurrent task. Moreover, shifts in decision criteria were found to be associated with the concurrency benefit effect.
\end{abstract}

Keywords Attentional blink · Multitasking - Detection sensitivity · Decision criterion - Temporal reproduction

Multitasking is most often highly taxing on human performance. A widely accepted notion is that information processing is constrained by a serial central bottleneck in such a way that there will be little flexibility in the deployment of attention (see Pashler,

R. Lapointe-Goupil · C. Fortin · B. Brisson · S. Tremblay $(\bowtie)$

École de Psychologie, Université Laval,

Québec G1K 7P4, Canada

e-mail: sebastien.tremblay@psy.ulaval.ca
1999). There are nevertheless reports, although scarce in the literature, of the beneficial effects of multitasking (see Navon \& Gopher, 1979). Such findings were recently obtained in studies combining the attentional blink (AB; Raymond, Shapiro, \& Arnell, 1992) procedure with various concurrent mental activities (Arend, Johnston, \& Shapiro, 2006; Olivers \& Nieuwenhuis, 2005, 2006; Taatgen, Juvina, Schipper, Borst, \& Martens, 2009). Such findings are rather surprising, given that the $\mathrm{AB}$ phenomenon is often viewed as a manifestation of the central bottleneck, which is thought to be at the root of multitasking deficits. As is detailed below, the present study was conducted to further investigate the nature of concurrency benefits in the $\mathrm{AB}$ by measuring response bias and detection sensitivity, in addition to report accuracy, while using a concurrent timing task known to require central resources continuously throughout the task.

The $\mathrm{AB}$ refers to the decline in accurate report of a second masked target (T2) when it is presented at short intertarget lags of about $200-500 \mathrm{~ms}$ after a first masked target (T1). In most common $\mathrm{AB}$ paradigms, the targets are embedded in a rapid serial visual presentation (RSVP) stream of distractors presented at fixation, and responses to both targets are made without speeded pressure, at the end of the RSVP stream. Thus, to be available for report, targets must be consolidated into short-term memory, where the representations must remain active until responses are made. Early bottleneck theories of the AB, such as the two-stage model (Chun \& Potter, 1995) and the central interference theory (Jolicœur, 1998, 1999), were based on the notion that central stages of processing have a limited capacity, allowing only a subset of information to proceed at any given time through 
these stages (see Pashler, 1999). Specifically, these theories proposed that when central attention mechanisms are engaged in consolidating a leading target (T1), they are not available to consolidate a subsequent target (T2). Consequently, T1 and T2 perceptual representations cannot be consolidated as efficiently at the same time as when $\mathrm{T} 1$ and $\mathrm{T} 2$ can be processed independently. While a representation of $\mathrm{T} 1$ is being consolidated into short-term memory for later report, consolidation of $\mathrm{T} 2$ has to wait (or undergo slower processing; see, e.g., Tombu \& Jolicœur, 2003), during which period the representation of T2 decays and is more susceptible to overwriting by a trailing distractor before being recoded in a more stable format that supports conscious report. It is interesting to note that when $\mathrm{T} 1$ and $\mathrm{T} 2$ are associated with identical tasks (e.g., identifying letter targets among digit distractors), it is not unusual to observe little or no decline in T2 accuracy when $\mathrm{T} 2$ is presented in the first serial position after T1 (i.e., at lag 1), a phenomenon known as lag 1 sparing (for a review, see Dell'Acqua, Jolicœur, Pascali, \& Pluchino, 2007; Visser, Bischof, \& Di Lollo, 1999). This hallmark effect of the $A B$ is often explained by the slow temporal closing of an attentional gate that governs the passage of perceptual representations to subsequent central stages of processing.

As was detailed in a recent review (Dux \& Marois, 2009), the role of central capacity limits in the production of the $\mathrm{AB}$ is accepted by the majority of extant theories (but see, e.g., Di Lollo, Kawahara, Shahab Ghorashi, \& Enns, 2005; Olivers \& Meeters, 2008). However, important doubt has been shed on the nature of these limits and, specifically, on the proposal that the $A B$ is caused by an inflexible structural bottleneck. A result that has significantly contributed to questioning the inflexible bottleneck model is the counterintuitive finding that the $\mathrm{AB}$ can be attenuated when performed under conditions of concurrent processing or distraction (Arend et al., 2006; Olivers \& Nieuwenhuis, 2005, 2006; Taatgen et al., 2009). Such concurrency benefits in the $\mathrm{AB}$ were first reported by Olivers and Nieuwenhuis (2005). They showed that the magnitude of the $\mathrm{AB}$ was reduced when participants performed the $\mathrm{AB}$ task concurrently with activities such as free association or monitoring a musical sequence for the occurrence of an occasional yell, as compared with when the $\mathrm{AB}$ task was performed alone. These concurrency benefits were also observed when participants were instructed to maintain a visual pattern in memory during the $\mathrm{AB}$ task or simply were asked to reduce their level of concentration on the $\mathrm{AB}$ task (Olivers \& Nieuwenhuis, 2006). Other labs have also reported concurrency benefits. Arend et al. showed concurrency benefits when a distracting visual background made of inward-moving, outwardmoving, or flickering dots was superimposed on the RSVP stream. Taatgen et al. also reported a reduced $\mathrm{AB}$ when the $\mathrm{AB}$ task was performed concurrently with a peripheral red dot detection task.

In order to account for these results, Olivers and Nieuwenhuis (2006) put forward the overinvestment hypothesis, which proposes that the $\mathrm{AB}$ may result from an excessive amount of resources devoted to the task of detecting and identifying targets embedded in an RSVP stream of distractors. In a similar way to bottleneck models, the overinvestment hypothesis assumes the existence of a capacity-limited postperceptual stage to which only a few items can gain access in order to be consolidated for further report. For an item to enter the capacity-limited stage, its activation must exceed an internal activation threshold. Inspired by the interference theory (e.g., Isaak, Shapiro, \& Martin, 1999; Shapiro \& Raymond, 1994), the overinvestment hypothesis assumes that an item's activation is influenced by similarity and temporal proximity to the target. Importantly, overall activation of items in the RSVP stream also increases as more attentional resources are devoted to the $\mathrm{AB}$ task. Consequently, allocating an excessive amount of resources to detecting and identifying targets in the RSVP stream could result in a greater number of distractors entering the capacity-limited stage, leading to more competition for encoding and, hence, more interference, which would result in the $\mathrm{AB}$ phenomenon, or at least modulate its amplitude. In contrast, if resources are diverted away from the RSVP stream, activation of distractors will be reduced, and therefore, fewer distractors will enter the capacity-limited stage, thus reducing competition/interference and, hence, the magnitude of the AB. This idea is compatible with Lavie's (2005) load theory of selective attention, according to which resources unneeded for targets spill over to distractors, thus creating interference.

The threaded cognition model (Taatgen et al., 2009) also regards the $\mathrm{AB}$ as a consequence of an overzealous strategy. According to the threaded cognition model, the $\mathrm{AB}$ arises from an overexertion of cognitive control when an intervening distractor is presented. To avoid a potential conflict between target detection and short-term consolidation in these conditions, an inhibitory signal suspends target detection while $\mathrm{T} 1$ is being consolidated, which leads to the AB. However, if cognitive control is diverted toward a concurrent task, the inhibitory signal is no longer sent (or is less efficient), which would explain the reduction or elimination of the $\mathrm{AB}$ under concurrent conditions. This proposal is compatible with the episodic simultaneous type, serial token model (Wyble, Bowman, \& Nieuwenstein, 2009), which proposes that an inhibitory feedback signal is sent during consolidation of a target to facilitate the formation of episodically distinct representations within short-term memory. 
Although the reported attenuation of the $\mathrm{AB}$ under concurrent conditions has been considered as strong evidence against bottleneck theories, other considerations suggest that such a conclusion may be premature. First, Olivers and Nieuwenhuis (2006) performed a meta-analysis across their experiments (Olivers \& Nieuwenhuis, 2005, 2006), which revealed a significant $3.4 \%$ improvement of $\mathrm{T} 1$ performance in the concurrent conditions, as compared with the single-AB conditions. According to the authors, this pattern of $\mathrm{T} 1$ performance is contrary to what bottleneck models would predict. However, if we assume that $\mathrm{T} 1$ accuracy reflects the efficiency of $\mathrm{T} 1$ processing, as recent electrophysiological evidence has shown (Brisson et al., 2010), bottleneck models would predict that an increase in T1 accuracy should be associated with a reduction of the $\mathrm{AB}$. This is because more efficient processing would create a shorter waiting period in which $\mathrm{T} 2$ has to wait before being consolidated, in the same way as when the difficulty of active short-term memory processes in the T1 task (Akyürek, Hommel, \& Jolicœur, 2007; Ouimet \& Jolicœur, 2007) or the difficulty of online response selection to T1 (see Jolicœur, Dell'Acqua, \& Crebolder, 1998, 2001, for reviews) is reduced. Note that even small increases in T1 accuracy, of less than 5\%, have been associated with a significant attenuation of the $\mathrm{AB}$ magnitude (Brisson, Spalek, \& Di Lollo, in press).

Second, failures to replicate concurrency benefits by adding music (see Olivers \& Nieuwenhuis, 2006, Note 1) or by using a distracting visual background (see Taatgen et al., 2009, p. 12) have been reported, shedding doubt on the reliability of the effect. More important, it can be argued that concurrent activities previously used to elicit concurrency benefits do not require central resources. For example, not only are the instructions to "concentrate less" or to "think about one's holiday" vague in terms of what kind of resources they involve, but also the compliance of participants in performing the task requirements cannot be measured, or assessed, unless we resort to a circular logic: That is, a concurrency benefit was observed, therefore the participants complied with the instructions; participants complied with the instructions, therefore a concurrency benefit was observed. The same criticism applies when distracting backgrounds were used as the concurrent distraction (Arend et al., 2006). In the case of experiments in which a measurable concurrent task was employed, evidence suggests that they may not involve central capacity. For example, an occasional yell (Olivers \& Nieuwenhuis, 2005), as well as a sudden change in color of a peripheral dot (Taatgen et al., 2009), can exogenously capture attention, and therefore detecting the presence of such targets does not necessarily require central attention mechanisms. Moreover, in relation to Olivers and Nieuwenhuis (2006, Experiment 1), in which the concurrent task required maintenance of a visual pattern in memory, it could be stated that previous research has suggested that maintenance in short-term memory - unlike consolidation - does not require active central resources (e.g., Jolicœur \& Dell'Acqua, 1998; Woodman \& Vogel, 2005).

Given the importance of concurrency benefits in the modeling of the $\mathrm{AB}$ in particular and of multitasking limitations in general, it is important to investigate concurrency effects using a concurrent task known to utilize central resources in a sustained fashion, which would reduce the amount of central capacity that could be devoted to consolidating targets in the AB task. Bottleneck models would predict that performing a central demanding concurrent task should inevitably increase the magnitude of the $\mathrm{AB}$. Therefore, if a beneficial or null effect on the $\mathrm{AB}$ is observed in these conditions, it would provide solid evidence in favor of strategic models, such as the overinvestment hypothesis (Olivers \& Nieuwenhuis, 2006) and the threaded cognition model (Taatgen et al., 2009), and against the inflexible structural bottleneck model (Chun \& Potter, 1995; Jolicœur, 1998, 1999). These results would be even more convincing if concurrency benefits in the $A B$ were observed in the absence of an effect of the concurrent task on T1 performance and if unrelated strategies, such as shifts in decision criteria, were controlled.

The first major goal of the present study was to investigate concurrency benefits in the $\mathrm{AB}$ while using a new concurrent task involving interval timing. Timing tasks are known to require attention continuously throughout the stimulus presentation, and they have been investigated extensively under concurrent-processing conditions. The results showed bidirectional interference between timing and numerous perceptual and cognitive tasks: Timing is affected by central demands in concurrent tasks, and performance in concurrent tasks is also perturbed by concurrent timing (see Brown, 1997, 2008, for reviews). The choice of a timing task as the concurrent task enabled us to investigate whether concurrency benefits in the $A B$ would be reproduced using a concurrent timing task, or whether concurrent timing would disturb visual detection in the $\mathrm{AB}$ paradigm, as results from timing studies suggest. We also added a single-timing condition, in addition to the single- $\mathrm{AB}$ and concurrent $\mathrm{AB}$-timing conditions, in order to evaluate concurrency effects in the timing task, as well as in the $\mathrm{AB}$ task.

In the concurrent-task condition, a time interval reproduction task was executed concurrently with the $\mathrm{AB}$ task. Participants were asked to estimate the duration of a tone that was presented throughout the RSVP stream used in the $\mathrm{AB}$ task and then to reproduce the duration of the tone (or RSVP stream, which was of the same length). Consequently, attention had to be devoted continuously to both the timing and the $\mathrm{AB}$ tasks. Numerous studies have shown 
that reproducing a time interval (here, the duration of the tone) requires central processing in dual-task paradigms, by demonstrating clear interference effects when time reproduction is performed with other concurrent tasks (Brown, 1995, 1997, 2006; Brown \& Merchant, 2007; Casini \& Macar, 1997; Champagne \& Fortin, 2008; Coull, Vidal, Nazarian, \& Macar, 2004; Field \& Groeger, 2004; Fortin \& Massé, 1999; Rammsayer \& Ulrich, 2005). Reproductions are usually more accurate (i.e., closer to the actual time intervals to be reproduced) when time reproduction is performed alone than in concurrent-task conditions. Assuming that attention must be allocated to the time estimation task during the RSVP in the present study, reproductions should be closer to the presented target intervals in the single-timing task than in concurrent-task conditions.

A second important objective of the present study was to evaluate whether concurrency benefits in the $A B$ could be explained by unrelated strategies, such as shifts in decision criteria. To evaluate the role of shifts in decision criteria in concurrency effects, we calculated, in addition to report accuracy, $A^{\prime}$ (an index of detection sensitivity) and $B^{\prime \prime}$ (an index of response bias, or decision criterion), borrowed from the signal detection theory (Green \& Swets, 1966; Wickens, 2002). Indexes of detection sensitivity have been applied previously to short-term memory (Murdock, 1965; Wilken \& Ma, 2004) and visual search (Eckstein, Thomas, Palmer, \& Shimozaki, 2000). Signal detection theory views detection as a probabilistic decision process about a given signal (the target). Appropriate stimulation builds up evidence for the signal up to a given threshold, which is determined by a decision criterion. Changes in the decision criterion can affect the way information is processed. For instance, a liberal decision criterion means that little evidence is needed to decide that a target was perceived, at the cost of a higher rate of false alarms (deciding that the target is present when it is absent). With a more conservative decision criterion, more evidence is necessary to judge that a target was perceived, which leads to a lower rate of false alarms. A high $A^{\prime}$ indicates a high probability of correct detection and a low probability of a false alarm. If concurrency benefits result from better detection sensitivity, the $\mathrm{AB}$ as measured with $A^{\prime}$ should be reduced in concurrent-processing conditions, relative to the single- $\mathrm{AB}$ condition. On the other hand, if adding a concurrent task does not increase detection sensitivity but, rather, produces a shift in decision criteria toward a liberal stance, we should not observe any concurrency benefits when analyzing $A^{\prime}$. With a more liberal criterion, participants need less evidence to decide that a target is present, which may lead to a higher percentage of correct detection (i.e., reduced $\mathrm{AB}$ ), but also to a higher percentage of false alarms. Detection and identification have been shown to produce
ABs of similar amplitude (e.g., Shapiro, Raymond, \& Arnell, 1994). Therefore, to measure detection sensitivity $\left(A^{\prime}\right)$ and response bias $\left(B^{\prime \prime}\right),{ }^{1}$ we presented T1 on half of the trials and $\mathrm{T} 2$ on half of the trials, and participants were required to report whether $\mathrm{T} 1$ was present or absent and whether $\mathrm{T} 2$ was present or absent.

Another means to assess the role of strategy in $\mathrm{AB}$ concurrency benefits is to investigate the effect of the order in which the participants performed the single- $A B$ and concurrent conditions. Taatgen et al. (2009) have shown that concurrency benefits were observed only when the single-AB block was performed before the concurrent block. When the order of blocks was reversed, the magnitude of the $\mathrm{AB}$ in the single- $\mathrm{AB}$ task was reduced, resulting in the absence of a concurrency benefit. If this block order effect is replicated here, and if shifts in decision criteria are also observed, but only when the single-AB block is performed before the concurrent block, it would provide further evidence linking shifts in decision criteria to the concurrency benefit effect. If shifts in decision criteria play no role in concurrency benefits, response bias should not be affected by block order, even though a block order effect is observed on concurrency benefit effects.

In summary, the main issue investigated in the present study was twofold. First, we sought to investigate whether previously reported concurrency benefits in the $\mathrm{AB}$ paradigm can also be observed with a concurrent timing task, which has been shown to require central capacity continuously throughout the presentation, or whether the usual interference effect of timing on concurrent tasks and of concurrent tasks on timing would be found. Second, we sought to evaluate whether the observed concurrency benefits resulted from increases in detection sensitivity or

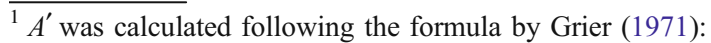

$A^{\prime}=0.5+\{[(H-F) *(1+H-F)] /[4 * H *(1-F)]\}$,

where $H$ is the hit rate (correctly reporting a target as present) and $F$ is the false alarm rate (reporting a target detection when the target is absent). If the false alarm rate was greater than the hit rate, the following formula was used (Aaronson \& Watts, 1987; Snodgrass \& Corwin, 1988):
}

$A^{\prime}=0.5-\{[(F-\mathrm{H}) *(1+F-H)] /[4 * F *(1-H)]\}$.

A measure of response bias, $B^{\prime \prime}$, was also calculated, following the formula by Grier (1971):

$B^{\prime \prime}=[H *(1-H)-F *(1-F)] /[H *(1-H)+F *(1-F)]$.

If the false alarm rate was greater than the hit rate, the following formula was used (Aaronson \& Watts, 1987; Snodgrass \& Corwin, 1988):

$B^{\prime \prime}=[F *(1-F)-H *(1-H)] /[H *(1-H)+F *(1-F)]$. 
rather, could be accounted for, at least in part, by changes in decision criterion. Mean time intervals reproduced under concurrent-task conditions and time reproduction singletask conditions were also contrasted. In Experiment 1a, a binary choice visual detection task was associated with $\mathrm{T} 1$ and $\mathrm{T} 2$, in which the first response involved indicating whether T1 was present or absent and the second response involved indicating whether $\mathrm{T} 2$ was present or absent. Experiment $1 \mathrm{~b}$ was designed to compute identification data in addition to detection data and signal detection theory indicators $\left(B^{\prime \prime}\right.$ and $\left.A^{\prime}\right)$. Participants had to decide first whether T1 and T2 were present or absent in the RSVP and then had to report the identity of the targets, when present. In Experiment 1b, target identification thus followed target detection. The goal of Experiment $1 \mathrm{~b}$ was to evaluate whether adding the requirement to identify the targets, in addition to detecting them, could have an impact on the response criterion participants adopted in the detection task. Indeed, it is possible that in these conditions, participants would respond that a target was present only if they were able to identify it and that this would impose a conservative criterion that could differentially impact performance in the single- $\mathrm{AB}$ and concurrent conditions. Because previous $\mathrm{AB}$ studies that investigated concurrency benefits required identification of both $\mathrm{T} 1$ and $\mathrm{T} 2$, we felt it was important to investigate the role of response bias in a context where $\mathrm{T} 1$ and $\mathrm{T} 2$ had to be identified.

\section{Method \\ Participants}

Sixty participants from Université Laval volunteered for this study. Half of these participants performed Experiment $1 \mathrm{a}$, and the other half performed Experiment 1b. One participant in Experiment 1a had to be excluded because of exceptionally low performance on the $\mathrm{AB}$ task. The remaining 59 participants were between 19 and 32 years of age. All participants reported normal hearing and normal or corrected-to-normal vision. They were paid $\$ 6$ for their participation.

\section{Stimuli and apparatus}

The experiment was run with E-Prime software on a PC computer. All visual stimuli (Arial 50 font) were white on a black background. Distractors were chosen randomly from among the capital letters of the alphabet, excluding A, B, I, $\mathrm{O}, \mathrm{Q}$, and $\mathrm{Z}$ to minimize target-distractor confusion. Targets were chosen without replacement from among the numbers 2, 3, 4, 7, 8, and 9. The first target, T1, was always the seventh stimulus in the stream. The second target, T2, was always different from the first target. In the absence of target- $\mathrm{T} 1, \mathrm{~T} 2$, or both - the target stimulus was replaced with one distractor.

\section{Procedure}

The experimental session lasted for approximately $70 \mathrm{~min}$. Participants were tested individually, at approximately 50 $\mathrm{cm}$ from a computer monitor. When ready, the participants initiated the trial by pressing the space bar on the computer keyboard. At keypress, a fixation cross $(+)$ was displayed for $500 \mathrm{~ms}$ at the center of the screen, followed by the presentation of the stimuli. As is illustrated in Fig. 1, there was an RSVP stream of uppercase letters and digits presented on the monitor screen, while an auditory stimulus, a continuous tone $(500 \mathrm{~Hz})$, was presented

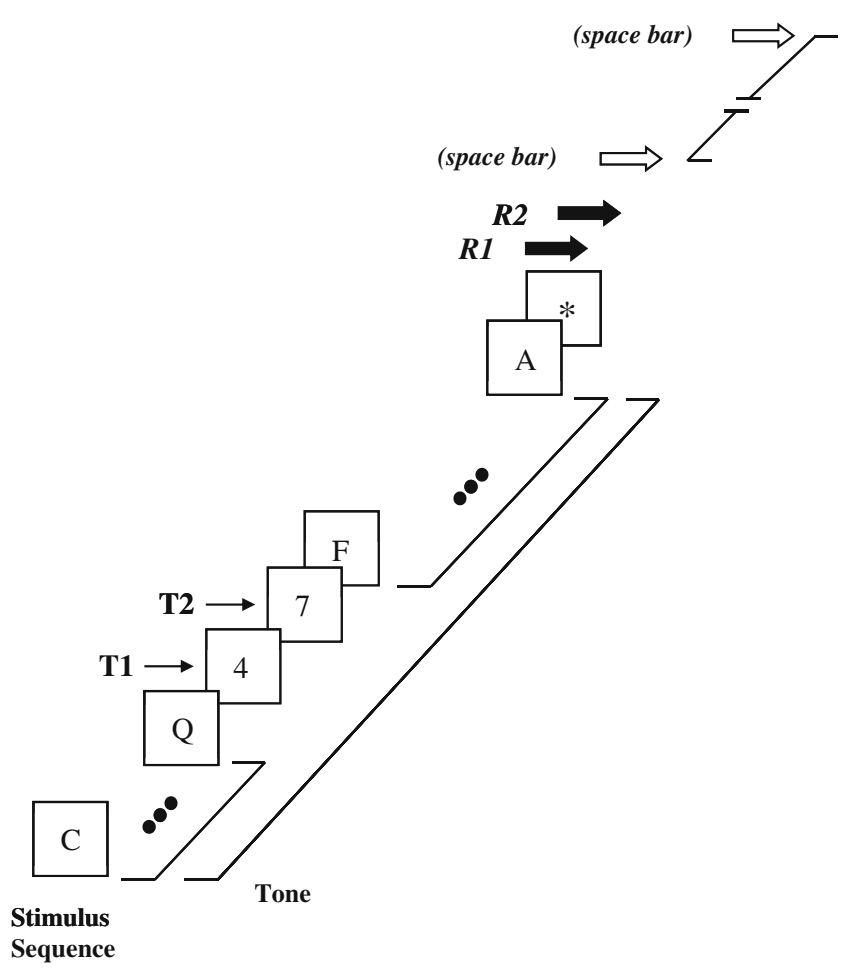

Fig. 1 Experiment 1: Example of trial in the concurrent-task condition. Twenty alphanumeric characters were successively presented ( $\mathrm{C}$ to $\mathrm{A}$ in the figure), among which two digits, T1 and T2 (4 and 7 in the figure), had to be reported as being present or absent among letter distractors. When present, T1 was always the 7th stimulus. When present, T2 could be in position 1, 2, 4, or 8 after $\mathrm{T} 1$ (it is in position 1 in the figure). R1 and R2 stand for "response to T1" and "response to T2." After executing these two responses, participants were asked, in the concurrent-task condition, to reproduce the duration of the tone by pressing the space bar twice. The tone was synchronized with the visual sequence presentation. In the single-ABtask condition, participants did not have to reproduce the tone duration, and in the single-temporal-task condition, participants did not have to execute R1 and R2. A more detailed description of experimental trials in the concurrent-task, single-AB-task, and singletemporal-task conditions is provided in the text 
simultaneously through headphones. The RSVP consisted of 20 letters and digits presented successively at a constant rate of 70,85 , or $100 \mathrm{~ms}$, with no interstimulus interval. The total duration of the visual sequence could therefore be $1,400,1,700$ or $2,000 \mathrm{~ms}$, depending on the rate of presentation. The visual sequence and tone presentation were synchronized so that they started and ended simultaneously; thus, the tone was the same duration as the RSVP. The distractors were uppercase letters, and the targets were digits. When present, the first target (T1: "4" in Fig. 1), was always in the seventh position. The 13 following stimuli could include a second target (T2) or not, which, when present, could be in position $1,2,4$, or 8 after T1. T2 is the "7" digit in Fig. 1 and is placed in position 1 because it follows T1 immediately. After the last stimulus, an asterisk (*) appeared, indicating the end of the visual sequence.

In the single-AB task condition of Experiment 1a, participants had to execute two responses, the first (R1) to indicate whether $\mathrm{T} 1$ was present or absent, and the second (R2) to indicate whether T2 was present or absent. R1 and R2 were executed by pressing either the " 1 " (present) or " 2 " (absent) key on the numerical keyboard of the computer. In Experiment 1b, participants had to report the identity of the targets that were reported as present by pressing the corresponding digit key on the computer keyboard. If a target was reported as absent, no response regarding the target identity was required. In both experiments, a fixation cross appeared after the last response, indicating the beginning of the next trial. Responses were not speeded.

In the concurrent-task condition, immediately after executing the last response in the $\mathrm{AB}$ task, participants had to reproduce the tone (or RSVP) duration by pressing the space bar twice, the first keypress indicating the beginning of the tone and the second keypress indicating when, according to the participant, the tone ended. In the single-temporal-task condition, participants were asked to look at the visual sequence but to ignore it and to pay attention to the tone duration only. In this condition, participants were not required to execute $\mathrm{R} 1$ and $\mathrm{R} 2$, but only to reproduce the time interval with the space bar when the asterisk appeared. Participants were informed that one or two targets could be presented on each trial. Temporal reproductions were measured to the nearest millisecond.

The experimental session was divided into three blocks in which the three experimental conditions were tested separately: a block of single-AB trials on which the $A B$ task was performed alone, a block of single-temporal-task trials on which temporal reproduction was performed alone, and a concurrent-task block in which the visual and temporal tasks were executed concurrently. All the participants were tested in the three blocks, and the order of the blocks was counterbalanced across participants.
The AB task in the single-AB-task condition was practiced in one 12-trial practice block, after which the corresponding 144-trial experimental block was completed. There were 3 trials in each combination of the following factor levels: duration of stimulus presentation (three values: $1,400,1,700$, or 2,000 ms), combination of T1-T2 presence or absence (four values: $\mathrm{T} 1$ and $\mathrm{T} 2$ present, $\mathrm{T} 1$ present and $\mathrm{T} 2$ absent, $\mathrm{T} 1$ absent and $\mathrm{T} 2$ present, and $\mathrm{T} 1$ and $\mathrm{T} 2$ absent), and intertarget lag, or serial position of T2 relative to $\mathrm{T} 1$ (first, second, fourth, or eight stimulus after T1, which defined positions 1, 2, 4, and 8, respectively). The single-temporal-reproduction task was first practiced on 12 practice trials (6 with feedback on temporal reproduction accuracy, followed by 6 with no feedback), which were followed by 30 experimental trials with no feedback. The three target durations to be reproduced, $1,400,1,700$, and $2,000 \mathrm{~ms}$, were reproduced ten times during the experimental trials. When feedback was provided on temporal reproduction accuracy in practice trials of temporal reproduction, a visual message appeared on the monitor screen, indicating to the participant whether his/her temporal reproduction was too short, too long, or correct. A "correct" temporal reproduction was a reproduction that was within a $10 \%$ temporal window centered on the target time interval (e.g., for the 1,400 -ms target interval, a reproduction shorter than 1,470 or longer than $1,330 \mathrm{~ms}$ ). Concurrent-task parameters were the same as those in the single-AB task, except that there were 16 practice trials on which the AB task and the temporal task were performed concurrently. On the first 8 practice trials, feedback on temporal reproduction accuracy was provided. In each block, values for all the factors were selected randomly on each trial, with the constraint that the number of trials was the same for each combination of factor levels. Feedback on report accuracy in the $\mathrm{AB}$ task was never provided, either on practice trials or on experimental trials.

\section{Results}

Separate analyses were conducted to assess performance in the $\mathrm{AB}$ task and in the timing task. Presentation rates were collapsed in the AB task analyses, and T1-T2 lag was collapsed in the timing task analyses. The Bonferroni adjustment for multiple comparisons was applied when appropriate. When the Mauchly test for sphericity was significant, the Greenhouse-Geisser correction was applied in all ANOVAs reported in the present study.

\section{AB task}

We assessed performance in the AB task using two measures, percentage of correct detection and detection 
sensitivity $\left(A^{\prime}\right)$, for both targets. Given that the $\mathrm{AB}$ is defined as a decline in accurate report of $\mathrm{T} 2$ when $\mathrm{T} 2$ is presented shortly after T1 (i.e., at short lags), only typical dual-target RSVP trials were used to calculate T1 detection accuracy, and of these trials, only $\mathrm{T} 1$ correct trials were used to calculate T2 detection accuracy. For T2, we also computed $B^{\prime \prime}$ as a measure of response bias, on the basis of data from all types of trials (no target, T1 only, T2 only, and $\mathrm{T} 1-\mathrm{T} 2$ trials).

To calculate $A^{\prime}$ to $\mathrm{T} 1$, correct detection of $\mathrm{T} 1$ was defined as detection accuracy of $\mathrm{T} 1$ on trials where both $\mathrm{T} 1$ and $\mathrm{T} 2$ were presented, and false alarms were defined as 1 $\mathrm{T} 1$ detection accuracy on trials where only $\mathrm{T} 2$ was presented. To calculate $A^{\prime}$ to $\mathrm{T} 2$, correct detection of $\mathrm{T} 2$ was defined as detection accuracy of $\mathrm{T} 2$ on trials where both $\mathrm{T} 1$ and $\mathrm{T} 2$ were presented, and false alarms were defined as 1 T2 detection accuracy on trials where only T1 was presented. Trials on which T1 was not correctly detected were rejected from the calculation of both correct

Fig. 2 T1 performance in Experiments 1a and 1b. Upper panels: Mean percentages of correct detection (left) and detection sensitivity (right: $A^{\prime}$ ) in Experiment 1a as a function of block order, block type, and lag. Lower panels: Mean percentages of correct detection (left), correct identification (center), and detection sensitivity (right: $A^{\prime}$ ) in Experiment $1 \mathrm{~b}$ as a function of block order, block type, and lag. Error bars represent the standard errors of the means detections and false alarms. Furthermore, on T1-only trials, $\mathrm{T} 1$ was always presented in the same position, and there was no lag, since T2 was absent. Consequently, all T1-only trials in a given block type were identical, and thus, lags were collapsed in the calculation of the false alarm rate of $\mathrm{T} 2$ detection. Also, false alarm rate was based on the same trials for each lag in a given block type. Contrary to T1only trials, however, T2-only trials differed between lag conditions, because $\mathrm{T} 2$ was not presented at the same position in different lag conditions. Therefore, the false alarm rate of $\mathrm{T} 1$ detection was based on different trials for each lag $\times$ block type cell.

The identification task was required only in Experiment $1 \mathrm{~b}$, and therefore, identification data were obtained only in this experiment.

T1 performance T1 performance on dual-task trials is plotted as a function of block type, block order, and T1T2 lag in Fig. 2. Data from Experiment 1a are presented in

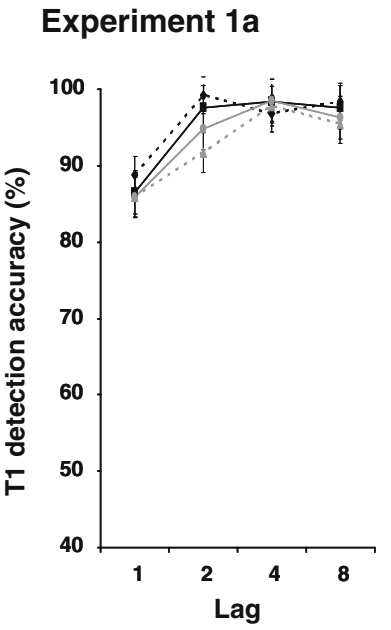

Experiment 1b

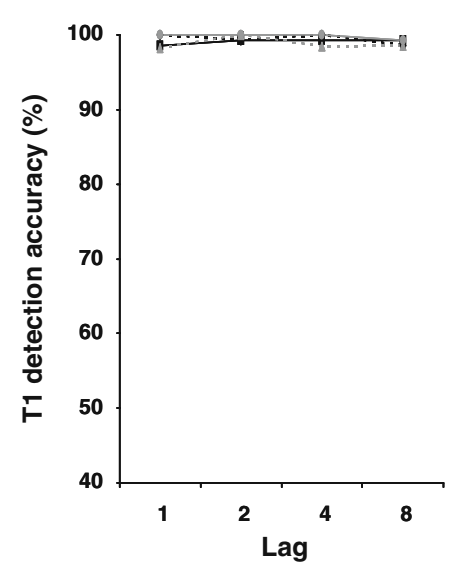

Group 1

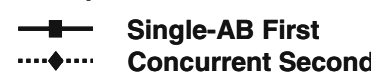

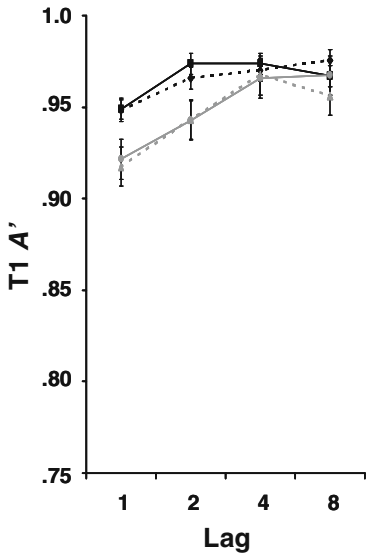
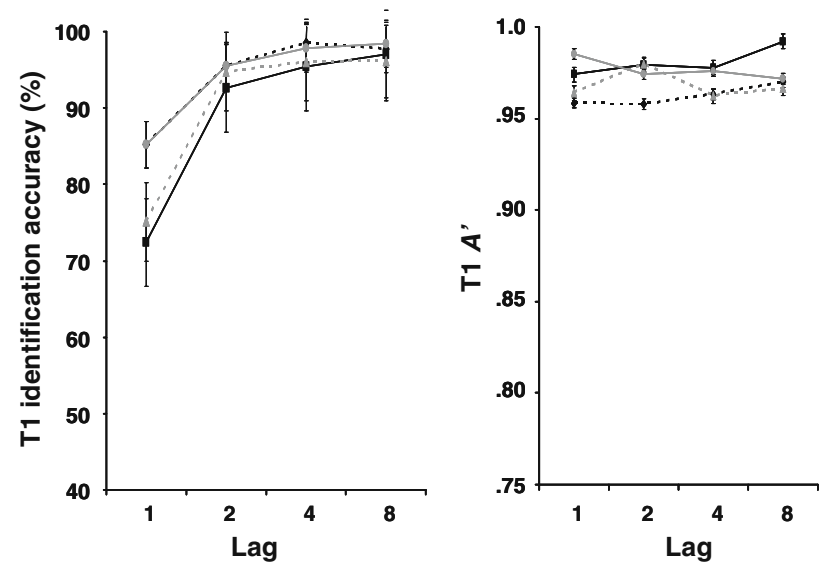

Group 2

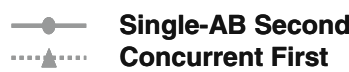


the upper panels, and data from Experiment $1 \mathrm{~b}$ are presented in the lower panels.

T1 detection accuracy $\mathrm{T} 1$ detection accuracy (see Fig. 2, upper left and lower left panels) was analyzed in a 2 (experiment: Experiment 1a, Experiment 1b) $\times 2$ (block order: single $\mathrm{AB}$ before concurrent, concurrent before single $\mathrm{AB}) \times 2$ (block type: single-AB task, concurrent task $) \times 4(\mathrm{~T} 1-\mathrm{T} 2$ lag: $1,2,4,8)$ repeated measures analysis of variance (ANOVA), with block type and T1-T2 lag as within-subjects factors and experiment and block order as between-subjects factors. T1 detection accuracy was generally higher in Experiment $1 \mathrm{~b}$ than in Experiment 1a, leading to a main effect of experiment, $F(1,55)=25.25$, $p<.001$. As has often been observed in $\mathrm{AB}$ studies, $\mathrm{T} 1$ accuracy was lower when $\mathrm{T} 2$ was presented at lag 1 than at other lags, leading to a main effect of lag, $F(3,165)=$ $15.78, p<.001$. However, the lag effect was absent in Experiment $1 \mathrm{~b}$, which explained the lag $\times$ experiment interaction, $F(3,165)=15.21, p<.001$ (and also the main effect of experiment). No other main effect or interaction was observed, all $F_{\mathrm{s}}<1.42$.

T1 identification accuracy $\mathrm{T} 1$ identification accuracy was lower when T2 was presented at lag 1 than at other lags (see Fig. 2, lower center panels), leading to a main effect of lag, $F(3,84)=44.11, p<.001$. No main effect of block type or block order was observed, both $F_{\mathrm{S}}<1$. However, both the block type $\times$ block order interaction, $F(1,28)=$ 13.04, $p=.001$, and the block type $\times$ lag $\times$ block order interaction, $F(3,84)=4.84, p=.015$, were significant, reflecting better performance at lag 1 in the second block than in the first block, independently of block type.

T1 detection sensitivity $\left(A^{\prime}\right)$ T1 detection sensitivity was also impaired at lag 1, as compared with the other lags, but only in Experiment 1a (see Fig. 2, upper left and lower left panels). This was evidenced by a main effect of lag, $F(3,165)=5.27, p=.004$, and a lag $\times$ experiment interaction, $F(3,165)=4.33, p=.011$. The main effect of experiment was also marginally significant, $F(1,55)=3.62$, $p>.06$. No other main effect or interaction was observed, all $F_{\mathrm{S}}<1.98$, except for the main effect of block type, $F(1,55)=3.09, p>.08$.

T2 performance T2 performance on dual-task trials is plotted as a function of block type, block order, and T1T2 lag in Fig. 3. Data from Experiment 1a are presented in the upper panels, and data from Experiment $1 \mathrm{~b}$ are presented in the lower panels.

$T 2 \mid T 1$ detection accuracy $\mathrm{T} 2 \mid \mathrm{T} 1$ detection accuracy is presented in the upper left and lower left panels for
Experiment 1a and Experiment 1b, respectively. The four-way interaction was not significant, $F(3,159)=$ $1.83, p>.15$. On the other hand, a block order $\times$ block type $\times$ lag interaction was significant, $F(3,159)=3.38$, $p=.027$, which led us to perform separate ANOVAs for participants who performed the single-AB task before the concurrent task and participants who performed the single$\mathrm{AB}$ task after the concurrent task. When the single- $\mathrm{AB}$ task was performed first, a main effect of lag was observed, $F(3,78)=7.53, p=.001$. Pairwise comparisons confirmed that T2|T1 accuracy was lower at lags 2 and 4 than at lag 8 , both $p \mathrm{~s}<.001$. This drop in T2|T1 accuracy at short lags is the signature of the $\mathrm{AB}$ deficit. Note that performance at lag 1 was similar to performance at lag 8 , $t(28)=0.89, p>.37$. This phenomenon is known as lag 1 sparing and is a hallmark of the $\mathrm{AB}$ phenomenon. A main effect of block was also observed, $F(1,26)=6.34, p=$ .018 , and the block $\times$ lag interaction was also significant, $F(3,78)=3.56, p=.033$. Paired sample $t$ tests confirmed that the average of the T2|T1 detection accuracy at lags 1 and 8 was significantly higher in the concurrent condition than in the single-AB-task condition, $t(28)=2.81, p=$ .009 , as was the average of the T2|T1 detection accuracy at lags 2 and $4, t(28)=5.71, p<.001$. Importantly, the size of the effect of block type was greater at lags 2 and 4 $(20.7 \%)$ than at lags 1 and $8(4.5 \%), t(28)=4.37, p<.001$, revealing the presence of a concurrency benefit effect in the magnitude of the $A B$ in this group of participants.

When the single-AB task was performed after the concurrent block, a main effect of lag was again observed, $F(3,87)=8.70, p<.001$. Pairwise comparisons showed that T2|T1 accuracy was lower at lags 2 and 4 than at lag 8 , both $p \mathrm{~s} \leq .01$. Performance at lag 1 was again similar to performance at lag $8, t(29)=0.89, p>.37$. Contrary to what was observed when the single-AB task was performed before the concurrent task, for participants who performed the single-AB task after the concurrent task, we observed no main effect of block, $F(1,29)=1.97, p>.17$, and no block $\times$ lag interaction, $F(3,87)=1.25, p>.29$. The absence of concurrency benefits in the $\mathrm{AB}$ was confirmed by subsequent paired-sample $t$ tests, which showed that the average of the T2|T1 detection accuracy at lags 2 and 4 was not significantly different between block type conditions, $t(29)=1.11, p>.27$, nor was the average of the T2|T1 detection accuracy at lags 1 and $8, t(29)=0.33, p>.74$.

T2|T1 identification accuracy T2|T1 identification accuracy in Experiment $1 \mathrm{~b}$ is presented in the lower center panel of Fig. 3. As for detection accuracy, a significant triple interaction was observed when T2|T1 identification accuracy was analyzed, $F(3,84)=3.14, p=.04$. When the single-AB task was performed before the concurrent task, a main effect of lag was observed, $F(3,42)=6.97, p=.001$. 
Fig. 3 T2 performance in Experiments 1a and 1b. Upper panels: Mean percentages of correct detection (left) and detection sensitivity (right: $A^{\prime}$ ) in Experiment 1a as a function of block order, block type, and lag. Lower panels: Mean percentages of correct detection (left), correct identification (center), and detection sensitivity (right: $A^{\prime}$ ) in Experiment $1 \mathrm{~b}$ as a function of block order, block type, and lag. Error bars represent the standard errors of the means
Experiment 1a

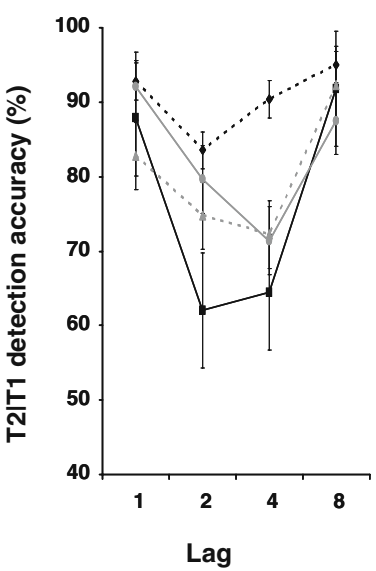

Experiment 1b

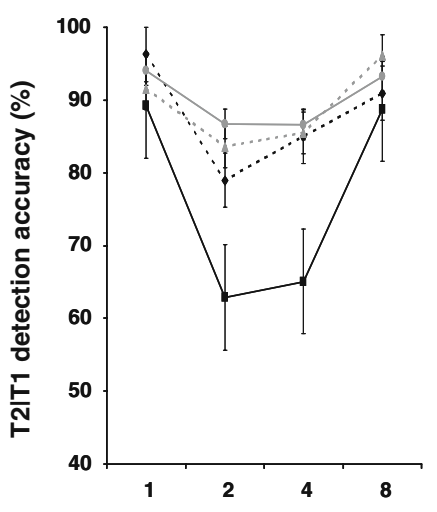

Lag
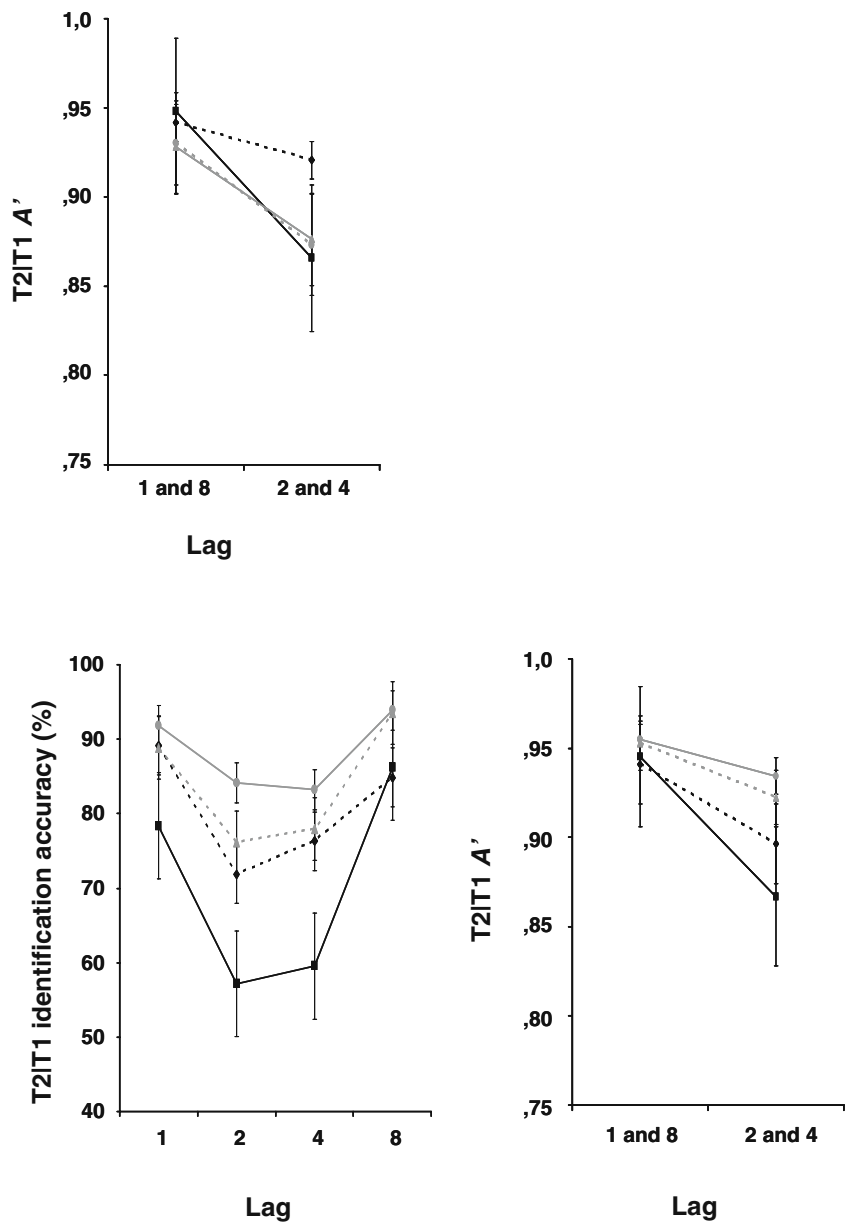

Group 1

$\begin{array}{ll}\longrightarrow \ldots & \text { Single-AB First } \\ \ldots \ldots & \text { Concurrent Second }\end{array}$
Group 2

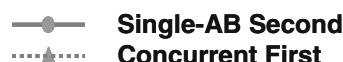

Single-AB Second
Concurrent First
Pairwise comparisons confirmed that $\mathrm{T} 2 \mid \mathrm{T} 1$ accuracy was lower at lags 2 and 4 than at lag 8 , both $p \mathrm{~s}<.04$, and performance at lag 1 was similar to performance at lag 8 , $p=1$. A main effect of block was also observed, $F(1,14)=$ $8.16, p=.013$, and the block $\times$ lag interaction was marginally significant, $F(3,42)=2.83, p=.066$. Pairedsample $t$ tests showed that the average of the T2|T1 identification accuracy at lags 1 and 8 was statistically equivalent in the concurrent condition to that in the singleAB-task condition, $t(14)=1.33, p>.20$, whereas the average of the T2|T1 identification accuracy at lags 2 and 4 was higher in the former than in the latter block type condition, $t(14)=2.85, p=.013$.

When the single-AB task was performed after the concurrent block, a main effect of lag was again observed, $F(3,42)=6.98, p=.003$, and pairwise comparisons confirmed that T2|T1 accuracy was lower at lags 2 and 4 than at lag 8 , both $p \mathrm{~s}<.05$, whereas performance at lag 1 was similar to performance at lag $8, p=1$. Contrary to what was observed when the single-AB task was performed before the concurrent task, here we observed no main effect of block, $F(1,14)=2.60, p>.12$, and no block $\times$ lag interaction, $F<1$. The absence of concurrency benefits in the $\mathrm{AB}$ was confirmed by subsequent paired-sample $t$ tests, which showed that the average of the T2|T1 identification accuracy at lags 1 and 8 was not significantly different between block type conditions, $t(14)=0.054, p>.60$, nor was the average of the T2|T1 identification accuracy at lags 2 and $4, t(14)=1.84, p=.088$.

$T 2 \mid T 1$ detection sensitivity $\left(A^{\prime}\right)$ In relation to T2 performance, the result of main interest was the magnitude of the $\mathrm{AB}$ (i.e., the difference in $\mathrm{T} 2$ performance during the $\mathrm{AB}$ period, as compared with outside the $\mathrm{AB}$ period) in the single- $\mathrm{AB}$ and concurrent conditions. In order to increase the stability of our measures of $A^{\prime}$ to T2, we collapsed hit rate data from lag 1 and lag 8 trials to assess performance outside the $\mathrm{AB}$ and collapsed hit rate data from lag 2 and 
lag 4 trials to assess performance during the $\mathrm{AB}$ (for similar methods, see Colzato, Spapé, Pannebakker, \& Hommel, 2007; Taatgen et al., 2009). As was mentioned above, the false alarm rate was calculated by collapsing all lags in a given block type. Therefore, for each participant, the hit rate was calculated on the basis of 18 trials for each experimental cell, and the false alarm rate was based on 36 trials. The $A^{\prime}$ values measured in Experiments $1 \mathrm{a}$ and $1 \mathrm{~b}$ are presented in the upper right and lower right panels of Fig. 3. They were analyzed in a 2 (experiment: Experiment 1a, Experiment 1b) $\times 2$ (block order: single $A B$ before concurrent, concurrent before single $\mathrm{AB}) \times 2$ (block type: single-AB task, concurrent-task) $\times 2$ (T1-T2 lag: 1 and 8,2 and 4) repeated measures ANOVA, with block type and T1-T2 lag as within-subjects factors and block order as a between-subjects factor. As for T2 detection accuracy, no main effect of experiment and no any interaction with this factor were observed, and therefore, the data from both experiments were collapsed in subsequent analyses. A significant triple interaction between block order, block type, and lag was observed for T2 detection sensitivity, $F(1,57)=9.35, p=.003$. For participants who performed the single- $\mathrm{AB}$ task before the concurrent task, a main effect of lag was observed, $F(1,28)=36.35, p<.001$, showing that the $\mathrm{AB}$ phenomenon was still present when both misses and false alarms were taken into account. The main effect of block type was also significant, $F(1,28)=4.66$, $p=.04$, as well as the block type $\times$ lag interaction, $F(1,28)=16.56, p<.001$. Paired-sample $t$ tests revealed a pattern of concurrency benefit in the $\mathrm{AB}$, in that the average of $A^{\prime}$ to T2 at lags 1 and 8 was not significantly different between block type conditions, $t(28)=0.65, p>.52$, but the average of $A^{\prime}$ to T2 at lags 2 and 4 was significantly higher in the concurrent condition than in the single-ABtask condition, $t(28)=3.50, p=.002$.

For participants who performed the single-AB task after the concurrent task, a main effect of lag on $\mathrm{T} 2$ detection sensitivity was again observed, $F(1,29)=18.04, p<.001$. No main effect of block type and no block $\times$ lag interaction were observed, both $F s<1$. The absence of concurrency benefits in the $A B$ in this group of participants was confirmed by subsequent paired-sample $t$ tests, which showed that the average of $A^{\prime}$ to T2 at lags 1 and 8 was not significantly different between block type conditions, $t(29)=0.27, p>.78$, nor was the average of $A^{\prime}$ to T2 at lags 2 and $4, t(29)=0.47, p>.64$.

Decision criteria $\left(B^{\prime \prime}\right) B^{\prime \prime}$ values were submitted to a 2 (experiment: Experiment 1a, Experiment 1b) $\times 2$ (block order: single $\mathrm{AB}$ before concurrent, concurrent before single $\mathrm{AB}$ ) $\times 2$ (block type: single-AB task, concurrent task) repeated measures ANOVA, with block type as a within-subjects factor and experiment and block order as between-subjects factors. There was no main effect of experiment, $F(1,55)=1.59, p>.21$, and no interaction (experiment $\times$ block order, $F<1$; experiment $\times$ block type, $F(1,55)=3.43, p=.07$; experiment $\times$ block order $\times$ block type, $F(1,55)=2.99, p>.08)$. Therefore, the data from both experiments were collapsed in subsequent analyses. The main effect of block order was not significant, $F<1$. However, a main effect of block type was observed, $F(1,57)=12.26, p=.001$, as well as a block order $\times$ block type interaction, $F(1,57)=11.43, p=.001$. Pairedsample $t$ tests confirmed that a shift in decision criterion was present for participants who performed the single- $A B$ task before the concurrent task, participants being more liberal in the concurrent-task block than in the single-ABtask block $\left(B^{\prime \prime}=-.02\right.$ and .42 in the concurrent-task and single-AB-task blocks, respectively), $t(28)=3.93, p=.001$. However, such a shift was absent for participants who performed the single $\mathrm{AB}$ task after the concurrent task $\left(B^{\prime \prime}=.17\right.$ and .17 in the concurrent-task and single-AB-task blocks, respectively), $t(29)=0.12, p>.90$.

\section{Timing task}

As is shown in Table 1 (left part), mean reproductions lengthened with increasing value of target durations to be reproduced in the single-temporal-task and concurrent-task conditions, $F(2,114)=817.58, p<.001$, confirming that the timing task was performed correctly in both conditions. Although this was true in both experiments, the lengthening of reproductions was slightly more pronounced in Experiment $1 \mathrm{~b}$ than in Experiment 1a, which led to an experiment $\times$ target duration interaction, $F(2,114)=3.54, p=.043$. The lengthening of reproductions with increasing target duration was more pronounced in the single-temporal-task than in the concurrent-task block, as shown by the significant interaction between block type and target duration, $F(2,114)=28.61, p<$ .001 . Indeed, when participants reproduced temporal intervals in single-temporal-task conditions, their temporal reproductions were closer to the actual presented durations than when the time reproduction task was performed in concurrent-task conditions. Finally, reproductions were generally longer in the concurrent-task block than in the single-temporal-task block, $F(1,57)=25.18, p<.001$, and this effect was larger in Experiment 1a than in Experiment $1 \mathrm{~b}$ (experiment $\times$ block type interaction, $F(1,57)=6.86$, $p=.011)$.

When block order (single AB first, concurrent first) was included as a between-subjects factor, there was no significant interaction with this factor, all $F \mathrm{~s}<1.37$, except for a block type $\times$ experiment $\times$ block order triple interaction, $F(1,55)=4.94, p=.03$. The main effect of block order was also marginally significant, $F(1,55)=$ 
Table 1 Means and standard deviations for reproduced intervals in Experiment 1a and Experiment 1b (in milliseconds), in each condition of block type (single temporal task, concurrent task) and at each value of target duration, for the group that performed the Single-AB task before the concurrent task (Group 1) and for the group that performed the Single-AB task after the Concurrent task (Group 2)

\begin{tabular}{|c|c|c|c|c|}
\hline \multirow[t]{2}{*}{ Condition } & \multicolumn{2}{|l|}{ Group 1} & \multicolumn{2}{|l|}{ Group 2} \\
\hline & $\begin{array}{l}\text { Single temporal } \\
M(S D)\end{array}$ & Concurrent & Single temporal & Concurrent \\
\hline Target duration & Experiment 1a & & & \\
\hline $1,400 \mathrm{~ms}$ & $1,507(90)$ & $1,698(157)$ & $1,503(141)$ & $1,830(203)$ \\
\hline $1,700 \mathrm{~ms}$ & $1,788(92)$ & $1,888(144)$ & $1,752(96)$ & $2,005(180)$ \\
\hline $2,000 \mathrm{~ms}$ & $1,964(105)$ & 2,045 (157) & $1,961(110)$ & 2,148 (219) \\
\hline Target duration & Experiment $1 b$ & & & \\
\hline $1,400 \mathrm{~ms}$ & $1,530(141)$ & $1,678(79)$ & $1,613(126)$ & $1,740(129)$ \\
\hline $1,700 \mathrm{~ms}$ & $1,788(150)$ & $1,921(114)$ & $1,879(115)$ & $1,944(159)$ \\
\hline $2,000 \mathrm{~ms}$ & $2,060(140)$ & $2,100(135)$ & $2,118(114)$ & $2,081(180)$ \\
\hline
\end{tabular}

$3.86, p=.055$, indicating that responses tended to be slower in the group of participants that performed the concurrent block first. In sum, these results indicate that the effect of block order on concurrency benefits in the AB is not caused by a trade-off between the temporal task and the AB task and, more generally, further support the absence of concurrency benefits when the concurrent task was performed first.

\section{Discussion}

A first objective of the present study was to verify whether concurrency benefits in the $\mathrm{AB}$, previously observed when the $\mathrm{AB}$ task was performed simultaneously with concurrent distracting activities (e.g., Olivers \& Nieuwenhuis, 2005, 2006), would be observed with a concurrent timing task that required central demands throughout the RSVP presentation. To provide a better description of the impact of adding a concurrent task, performance in the AB task was analyzed with multiple indicators, such as percentage of correct detection and percentage of correct identification, but also measures of detection sensitivity $\left(A^{\prime}\right)$ and decision criteria $\left(B^{\prime \prime}\right)$ borrowed from signal detection theory.

The pattern of results was very similar in conditions where the $\mathrm{AB}$ task required only detecting the targets (Experiment 1a) or required reporting the identity of the targets when they were reported as present (Experiment $1 b)$, despite the fact that $B^{\prime \prime}$ values were generally (but not statistically) higher in Experiment $1 \mathrm{~b}$ than in Experiment 1a. Higher $B^{\prime \prime}$ values in Experiment $1 \mathrm{~b}$ would suggest that the requirement of identifying targets in the $\mathrm{AB}$ task resulted in participants' adopting a more conservative response criterion, which could reflect a tendency to report that T2 was present only if the target was identified. This hypothesis is supported by the observation that, when a correct detection was reported, participants almost always correctly identified T2 (mean T2 identification accuracy given T2 correct detection was $96.3 \%$ ).
As was expected, the analyses of $\mathrm{T} 2$ performance on dual-target trials revealed an $\mathrm{AB}$ independently of whether correct detection, correct identification, or $A^{\prime}$ was used as the independent variable. Moreover, the amplitude of the $\mathrm{AB}$ in detection and identification was equivalent, replicating previous studies (e.g., Shapiro et al., 1994).

For participants who performed the single-AB block before the concurrent block, the $\mathrm{AB}$ was attenuated when the $\mathrm{AB}$ task was performed concurrently with the timing task, as compared with when it was performed alone. These results replicate concurrency benefits observed in previous studies (Arend et al., 2006; Olivers \& Nieuwenhuis, 2005, 2006; Taagen et al., 2009) using a measurable concurrent task known to require central resources. Results from the timing task demonstrated that participants performed this task adequately and that central attention mechanisms were drawn to the task as required. These concurrency benefits on T2 performance were obtained even though T1 performance was not modulated by the concurrent task. This is an important result because any concurrency benefit in the $\mathrm{AB}$ accompanied by an increase in $\mathrm{T} 1$ performance could be accounted for by bottleneck models, as explained in the Introduction. Moreover, any concurrency benefit in the $\mathrm{AB}$ accompanied by a decline in $\mathrm{T} 1$ performance could be accounted for by a trade-off between T1 and T2, which could also be explained by traditional capacity-based models.

Interestingly, for participants who performed the single$\mathrm{AB}$ condition before the concurrent condition, the $B^{\prime \prime}$ indicator suggests that these participants who performed the single-AB task before the concurrent task became more liberal in their decision to report the presence of $\mathrm{T} 2$ under concurrent-task conditions, resulting in higher rates of false alarms in T1-only trials, when T2 was not presented. Although shifts in response criterion cannot explain the whole of the effect, because concurrency benefits were also observed when both correct detection and false alarms were taken into account using the $A^{\prime}$ measure (and with the 
identification data of Experiment 1b), it nevertheless seems to play a significant role. The importance of the decision criteria in understanding concurrency benefit effects is highlighted by the fact that participants who performed the concurrent task before the single-AB task did not exhibit any shifts in decision criteria or any concurrency benefit.

The observation that concurrency effects were present for participants who performed the single-AB condition before the concurrent condition, but not for participants who performed the conditions in reverse order, replicates the block order effect reported in Taatgen et al. (2009). In addition, the present results suggest that the differences in shifts of response criteria, depending on which of the single-AB or concurrent task was performed first, participate in the block order effect. This suggests that participants are somewhat constrained to adopt a liberal bias in the concurrent condition. When the concurrent task is performed first, the bias is carried over to the next block. It may also be argued that practice increases detection sensitivity and/or temporal identification, but only in conditions where the single-AB task is performed alone first and then incorporated into a concurrent condition. In the present study, it is difficult to dissociate practice effects from the concurrency benefit effect. However, previous studies have failed to show practice effects in the AB within 400 trials when T2 was in the same color as all other items in the RSVP stream (e.g., Choi \& Watanabe, 2009, 2010), as in the present study, suggesting that practice effects cannot explain the reported block order effect (but see Taatgen et al., 2009).

Interestingly, temporal reproduction was not subject to a block order effect, suggesting that concurrency benefits are not caused by a trade-off between the temporal task and the AB task. Moreover, temporal reproduction lengthened with increases in the duration of the target intervals to be reproduced in the single-temporal-task condition, as well as in the concurrent-task condition. This is a key result in the analysis of timing performance, because it shows that the temporal task was performed correctly in both conditions, ensuring that attentional resources were allocated to the temporal task. Indeed, the objective of Experiment 1 being to test whether the $\mathrm{AB}$ task is performed better in concurrent central-processing conditions, it was essential to demonstrate that central resources were allocated to the concurrent task. The fact that participants discriminated well the three target durations, reproducing them in an orderly fashion in the single-temporal-task condition, as well as in the concurrent-task condition, confirms that during the simultaneous visual and auditory stimulus presentations, they were indeed processing information in order to reproduce their durations. The lengthening of temporal reproductions with increases in the target duration to be reproduced was more pronounced in the singletemporal-task than in the concurrent-task conditions. In fact, reproductions were closer to the presented interval in the single-temporal-taskcondition than in the concurrenttask condition. This result may be expected, given that timing is usually more accurate when performed alone than when performed under concurrent-processing conditions (e.g., Brown, 1995).

Reproduced intervals were also generally longer in the concurrent-task condition than in the single-temporal-task condition. This result may be taken to suggest that the participants based their temporal judgments on the visual presentation and that, in addition to its duration, the number of perceived changes in the visual presentation influenced temporal estimates (see, e.g., Brown, 1995). A similar observation was made in comparable conditions, in which participants were asked to reproduce target intervals of about 5 s (Macar \& Ivry, 2002). In Macar and Ivry's study, changes in visual stimuli had to be detected during 5-s intervals. Reproductions of this 5-s target interval lengthened as the number of changes increased during the interval, an effect that was specifically observed under conditions of higher load in the visual task. The authors interpreted this result as suggesting that participants used the number of perceived changes in visual presentation to estimate time when the number of changes to process in the visual task was high. When this mode of time estimation is used, nontemporal information, such as the perceived number of the events taking place during the target interval, is assumed to serve as the basis for inferring the passage of time (Brown, 1995; Hicks, Miller, \& Kinsbourne, 1976; Macar \& Ivry, 2002; McClain, 1983; Ornstein, 1969; Zakay \& Block, 2004; Zakay, Tsal, Moses, \& Shahar, 1994). Therefore, the number of perceived changes contributed to temporal judgments under these conditions, like the target interval itself, to which the temporal judgment was clearly related. In the present study, the number of perceived events during the target interval to be reproduced was likely to be greater in the concurrent-task than in the single-task condition: Participants had to process the visual stimuli in concurrent-task conditions, whereas they were instructed to ignore the RSVP in the single-temporal-reproduction task. This should have led to a higher number of perceived events during the interval to be reproduced in the concurrent-task condition than in the single-temporal-task condition, resulting in longer interval reproductions. In the concurrent condition, the number of visual stimuli, in addition to their duration, would therefore have contributed to determining the value of temporal estimates.

Although the difference in reproduced duration between the single-temporal-task and the concurrent-task conditions yields insightful (yet speculative) information regarding processes involved in the timing task in Experiment 1, the 
most important result in timing data with regard to our research endeavor is that temporal reproductions are clearly related to the target intervals to be reproduced in the concurrent-task condition. This demonstrates that while participants are performing the $\mathrm{AB}$ task, they also devote resources to the timing task, which is likely to reduce the amount of attention that participants can devote to the $\mathrm{AB}$ task. Despite this decrease in resources allocated to the $A B$ task, the $\mathrm{AB}$ was attenuated in the concurrent condition, replicating the concurrency benefits reported in previous studies (Olivers \& Nieuwenhuis, 2005, 2006; see also Arend et al., 2006; Taatgen et al., 2009).

Importantly, the finding that a central demanding concurrent task does not increase the magnitude of the $\mathrm{AB}$ and tends to result in an opposite, beneficial effect, even when shifts in decisional processes are controlled, does not fit well with the idea that attentional resources are limited and deployed in a serial manner, strictly constrained by an inflexible structural bottleneck (Chun \& Potter, 1995; Jolicœur, 1998, 1999). Indeed, structural bottleneck models would predict that diverting central attention toward the central demanding timing task would negatively affect consolidation in short-term memory of both $\mathrm{T} 1$ and $\mathrm{T} 2$, resulting in a larger $\mathrm{AB}$, but also likely in poorer $\mathrm{T} 1$ performance. Interestingly, T1 performance (independently of whether it was assessed using detection accuracy, identification accuracy, or $A^{\prime}$ ) was unaffected by the additional requirement of performing the concurrent timing task. This is an important result, because it rules out the possibility that participants mobilized additional resources to adequately perform the requirements of the more demanding concurrent-task condition. According to bottleneck models, mobilizing additional central resources could increase the efficiency of $\mathrm{T} 1$ processing, which not only would increase $\mathrm{T} 1$ performance, but also consequently would reduce the period $\mathrm{T} 2$ would have to wait before being consolidated itself and, thus, would result in attenuation of the $\mathrm{AB}$. On the other hand, a decrease in T1 performance would have provided evidence of a tradeoff between $\mathrm{T} 1$ and $\mathrm{T} 2$, which could be viewed as a consequence of capacity sharing (e.g., Shapiro, Schmitz, Martens, Hommel, \& Schnitzler, 2006; Tombu \& Jolicœur, 2003). The hypothesis that the concurrent task leads to a trade-off between $\mathrm{T} 1$ and $\mathrm{T} 2$ performance in the $\mathrm{AB}$ task is further denied by the observation that $\mathrm{T} 1$ performance was equivalent for participants who performed the single- $\mathrm{AB}$ condition before the concurrent condition and for participants who performed the conditions in reverse order. Despite equivalent $\mathrm{T} 1$ performance, only the former group exhibited a concurrency benefit effect. Capacity sharing could also assume that concurrency benefits could be the consequence of allocating more capacity to the $\mathrm{AB}$ task and less to the timing task, leading to better performance in the former task and poorer performance in the latter. However, block order (i.e., whether the single-AB task was performed before or after the concurrent task) had no effect on performance in the timing task and a strong effect on concurrency benefits, which is hard to reconcile with the capacity-sharing account. Moreover, the capacity-sharing account would predict that both $\mathrm{T} 1$ and $\mathrm{T} 2$ performance should improve in the concurrent task, which was not the case.

Instead, the fact that the $\mathrm{AB}$ was facilitated to some extent when performed with a time reproduction task supports models that assume that the $\mathrm{AB}$ is a consequence of an overinvestment of resources in the $\mathrm{AB}$ task (Olivers \& Nieuwenhuis, 2006) or an overexertion of cognitive control (Taatgen et al., 2009). For example, the overinvestment hypothesis suggested by Olivers and Nieuwenhuis (2006) proposed that when performed alone, with no concurrent task, a suboptimal mode of stimulus processing would be used in the $\mathrm{AB}$ paradigm, characterized by an unnecessary and excessive amount of attentional resources devoted to the RSVP. The excessive amount of attentional resources allocated to the RSVP stream promotes the entry of task-irrelevant items (i.e., distractors) into short-term memory, increasing processing interference in this second stage. Drawing away some resources from the RSVP by various manipulations promoting divided attention improves performance by reducing the number of taskirrelevant items that enter short-term memory. Previous manipulations include viewing a visual background unrelated to the $\mathrm{AB}$ task or pictures of positive affective content, asking participants to perform an additional memory task or a dot task or simply asking them to focus less on the $\mathrm{AB}$ task (Arend et al., 2006; Olivers \& Nieuwenhuis, 2005, 2006; Taatgen et al., 2009). The present study extends these conclusions to a time reproduction task, which is known to require central attention, but improved, or did not disturb, performance on the $A B$ task, depending on which of the single $\mathrm{AB}$ or concurrent tasks was performed first. The temporal reproduction task may have diverted central attention away from the AB task, thus reducing the amount of task-irrelevant information entering short-term memory that could interfere with second-stage processing of task-relevant target information, as postulated by the overinvestment hypothesis. The present study further suggests that a more liberal criterion in the $\mathrm{AB}$ task may interact with this relatively diffuse mental state, contributing to the more efficient mode of processing. These conclusions rely on the notion that participants performed the timing task in both experiments. Results show that the reproduced intervals varied with presented target durations and were obviously related to the real presented durations to be reproduced, confirming that resources were allocated to the timing task. This is a key result because it ensures that the participants were allocat- 
ing resources to the temporal task while they were performing the AB task.

Evidence of concurrency benefits reported here and in previous studies (Arend et al., 2006; Olivers \& Nieuwenhuis, 2005, 2006; Taatgen et al., 2009) provide support for models that advocate flexibility in attention limitations, such as Olivers and Nieuwenhuis (2006) overinvestment hypothesis and the threaded cognition model (Taatgen et al., 2009). The overinvestment hypothesis postulates that if an excessive amount of attentional resources are allocated to the RSVP stream, increased interference in the second stage of processing may result in the $A B$ phenomenon. However, if resources are diverted away from the RSVP, distractors are processed to a lesser extent, which reduces the magnitude of the $\mathrm{AB}$ phenomenon. This idea is compatible with Lavie's (2005) load theory of selective attention, according to which resources not needed for targets spill over to distractors, thus creating interference. On the other hand the threaded cognition model assumes that the AB is caused by an overzealous mechanism that sends an inhibition signal when a distractor is presented during the consolidation of $\mathrm{T} 1$, in order to protect $\mathrm{T} 1$. Increasing the load of the procedural module that controls the inhibition signals, by adding a concurrent task, will decrease the probability that the signal will be sent, thus reducing the AB. Whether it is through the overinvestment of attentional resources (Olivers \& Nieuwenhuis, 2006) or through an overexertion of cognitive control (Taatgen et al., 2009), attentional limitations seem to be flexible, rather than structural.

Authors Note This research was supported by research grants to Claudette Fortin and Sébastien Tremblay (Fonds Québécois de Recherche sur la Nature et les Technologies) and by a scholarship from the National Science and Engineering Research Council of Canada (NSERC) to Richard Lapointe-Goupil and a postdoctoral fellowship from NSERC to Benoit Brisson. We thank Josée Bluteau and Cindy Chamberland for their assistance in data collection. Correspondence concerning this article may be addressed to Sébastien Tremblay, École de Psychologie, Université Laval, Québec, QC, G1K 7P4 Canada, email: sebastien.tremblay@psy.ulaval.ca.

\section{References}

Aaronson, D., \& Watts, B. (1987). Extensions of Grier's computational formulas for $\mathrm{A}^{\prime}$ and $\mathrm{B}^{\prime \prime}$ to below-chance performance. Psychological Bulletin, 102, 439-442.

Akyürek, E. G., Hommel, B., \& Jolicœur, P. (2007). Direct evidence for a role of working memory in the attentional blink. Memory \& Cognition, 35, 621-627.

Arend, I., Johnston, S., \& Shapiro, K. L. (2006). Task-irrelevant visual motion and flicker attenuate the attentional blink. Psychonomic Bulletin \& Review, 13, 600-607.

Brisson, B., Robitaille, N., Deland-Bélanger, A., Spalek, T. M., Di Lollo, V., \& Jolicœur, P. (2010). Backward masking during rapid serial visual presentation affects the amplitude but not the latency of the P3 event-related potential. Psychophysiology, 47, 942-948.

Brisson, B., Spalek, T. M., \& Di Lollo, V. (in press). On the role of intervening distractors in the attentional blink. Attention, Perception, \& Psychophysics.

Brown, S. W. (1995). Time, change, and motion: The effects of stimulus movement on temporal perception. Perception \& Psychophysics, 57, 105-116.

Brown, S. W. (1997). Attentional resources in timing: Interference effects in concurrent temporal and nontemporal working memory tasks. Perception \& Psychophysics, 59, 1118-1140.

Brown, S. W. (2006). Timing and executive functions: Bidirectional interference between concurrent temporal production and randomization tasks. Memory \& Cognition, 34, 1464-1471.

Brown, S. W. (2008). Time and attention: Review of the literature. In S. Grondin (Ed.), Psychology of time (pp. 111-138). Bingley: Emerald Group Publishing.

Brown, S. W., \& Merchant, S. M. (2007). Processing resources in timing and sequencing tasks. Perception \& Psychophysics, 69, 439-449.

Casini, L., \& Macar, F. (1997). Effects of attention manipulation on perceived duration and intensity in the visual modality. Memory \& Cognition, 25, 812-818.

Champagne, J., \& Fortin, C. (2008). Attention sharing during timing: Modulation by processing demands of an expected stimulus. Perception \& Psychophysics, 70, 630-639. doi:10.3758/ PP.70.4.630

Choi, H., \& Watanabe, T. (2009). Perceptual learning: High-level influences. Learning with attention eliminates attentional blink on a long-term basis. Journal of Vision, 9, 856. doi:10.1167/9.8.856

Choi, H., \& Watanabe, T. (2010). Perceptual learning: Mechanisms and models. Changes induced by attentional training - capacity increase vs. allocation changes. Journal of Vision, 10, 1099. doi:10.1167/10.7.1099

Chun, M. M., \& Potter, M. C. (1995). A two-stage model for multiple target detection in rapid serial visual presentation. Journal of Experimental Psychology: Human Perception and Performance, 21, 109-127. doi:10.1037/0096-1523.21.1.109

Colzato, L. S., Spapé, M. M. A., Pannebakker, M. M., \& Hommel, B. (2007). Working memory and the attentional blink: Blink size is predicted by individual differences in operation span. Psychonomic Bulletin \& Review, 14, 1051-1057.

Coull, J. T., Vidal, F., Nazarian, B., \& Macar, F. (2004). Functional anatomy of the attentional modulation of time estimation. Science, 303, 1506-1508.

Dell'Acqua, R., Jolicœur, P., Pascali, A., \& Pluchino, P. (2007). Shortterm consolidation of individual identities leads to lag-1 sparing. Journal of Experimental Psychology: Human Perception and Performance, 33, 593-609. doi:10.1037/0096-1523.33.3.593

Di Lollo, V., Kawahara, J., Shahab Ghorashi, S. M., \& Enns, J. T. (2005). The attentional blink: Resource depletion or temporary loss of control? Psychological Research, 69, 191-200. doi:10.1007/s00426-004-0173-x

Dux, P. E., \& Marois, R. (2009). The attentional blink: A review of data and theory. Attention, Perception, \& Psychophysics, 71, 1683-1700. doi:10.3758/APP.71.8.1683

Eckstein, M. P., Thomas, J. P., Palmer, J., \& Shimozaki, S. S. (2000). A signal detection model predicts the effects of set size on visual search accuracy for feature, conjunction, triple conjunction, and disjunction displays. Perception \& Psychophysics, 62, 425-451.

Field, D. T., \& Groeger, J. A. (2004). Temporal interval production and short-term memory. Perception \& Psychophysics, 66, 808819.

Fortin, C., \& Massé, N. (1999). Order information in short-term memory and time estimation. Memory \& Cognition, 27, 54-62.

Green, D. M., \& Swets, J. A. (1966). Signal detection theory and psychophysics. New York: Wiley. 
Grier, J. B. (1971). Nonparametric indexes for sensitivity and bias: Computing formulas. Psychological Bulletin, 75, 424-429. doi: $10.1037 / \mathrm{h} 0031246$

Hicks, R. E., Miller, G. W., \& Kinsbourne, M. (1976). Prospective and retrospective judgments of time as a function of amount of information processed. The American Journal of Psychology, 89, 719-730. doi:10.2307/1421469

Isaak, M. I., Shapiro, K. L., \& Martin, J. (1999). The attentional blink reflects retrieval competition among multiple rapid serial visual presentation items: Tests of an interference model. Journal of Experimental Psychology: Human Perception and Performance, 25, 1774-1792. doi:10.1037/0096-1523.25.6.1774 and doi: $10.1037 / 0096-1523.26 .2 .479$

Jolicœur, P. (1998). Modulation of the attentional blink by on-line response selection: Evidence from speeded and unspeeded task decisions. Memory \& Cognition, 26, 1014-1032.

Jolicœur, P. (1999). Dual-task interference and visual encoding. Journal of Experimental Psychology: Human Perception and Performance, 25, 596-616. doi:10.1037/0096-1523.25.3.596

Jolicœur, P., \& Dell'Acqua, R. (1998). The demonstration of shortterm consolidation. Cognitive Psychology, 36, 138-202. doi:10.1006/cogp.1998.0684

Jolicœur, P., Dell'Acqua, R., \& Crebolder, J. (1998). Multitasking performance deficits: Forging some links between the $\mathrm{AB}$ and the PRP. In S. Monsell \& J. Driver (Eds.), Attention and performance XVIII: Control of cognitive performance (pp. 309-330). Cambridge: MIT.

Jolicœur, P., Dell'Acqua, R., \& Crebolder, J. M. (2001). The attentional blink bottleneck. In K. L. Shapiro (Ed.), The limits of attention: Temporal constraints in human information processing (pp. 82-99). Oxford: Oxford University.

Lavie, N. (2005). Distracted and confused? Selective attention under load. Trends in Cognitive Sciences, 9, 75-82. doi:10.1016/j. tics.2004.12.004

Macar, F., \& Ivry, R. (2002). Exploring the limits of the internal timer in prospective temporal judgments. Cognitive Processing, 3, 77-84.

McClain, L. (1983). Interval estimation: Effect of processing demands on prospective and retrospective reports. Perception \& Psychophysics, 34, 185-189.

Murdock, B. B., Jr. (1965). Signal-detection theory and short-term memory. Journal of Experimental Psychology, 70, 443-447. doi: $10.1037 / \mathrm{h} 0022543$

Navon, D., \& Gopher, D. (1979). On the economy of the humanprocessing system. Psychological Review, 86, 214-255. doi:10.1037/0033-295X.86.3.214

Olivers, C. N. L., \& Meeter, M. (2008). A boost and bounce theory of temporal attention. Psychological Review, 115, 836-863. doi:10.1037/a0013395

Olivers, C. N. L., \& Nieuwenhuis, S. (2005). The beneficial effect of concurrent task-irrelevant mental activity on temporal attention. Psychological Science, 16, 265-269. doi:10.1111/j.09567976.2005.01526.x

Olivers, C. N. L., \& Nieuwenhuis, S. (2006). The beneficial effects of additional task load, positive affect, and instruction on the attentional blink. Journal of Experimental Psychology: Human Perception and Performance, 32, 364-379. doi:10.1037/0096-1523.32.2.364

Ornstein, R. E. (1969). On the experience of time. Harmondsworth: Penguin.
Ouimet, C., \& Jolicœur, P. (2007). Beyond task-1 difficulty: The duration of $\mathrm{T} 1$ encoding modulates the attentional blink. Visual Cognition, 15, 290-304. doi:10.1080/13506280600693741

Pashler, H. E. (1999). The psychology of attention. Cambridge: MIT.

Rammsayer, T., \& Ulrich, R. (2005). No evidence for qualitative differences in the processing of short and long temporal intervals. Acta Psychologica, 120, 141-171. doi:10.1016/j. actpsy.2005.03.005

Raymond, J. E., Shapiro, K. L., \& Arnell, K. M. (1992). Temporary suppression of visual processing in an RSVP task: An attentional blink ? Journal of Experimental Psychology: Human Perception and Performance, 18, 849-860. doi:10.1037/0096-1523.18.3.849

Shapiro, K. L., \& Raymond, J. E. (1994). Temporal allocation of visual attention. In D. Dagenbach \& T. H. Carr (Eds.), Inhibitory processes in attention, memory, and language (pp. 151-188). San Diego: Academic.

Shapiro, K. L., Raymond, J. E., \& Arnell, K. M. (1994). Attention to visual pattern information produces the attentional blink in rapid serial visual presentation. Journal of Experimental Psychology: Human Perception and Performance, 20, 357-371. doi:10.1037/ 0096-1523.20.2.357

Shapiro, K. L., Schmitz, F., Martens, S., Hommel, B., \& Schnitzler, A. (2006). Resource sharing in the attentional blink. NeuroReport, 17, 163-166. doi:10.1097/01.wnr.0000195670.37892.1a

Snodgrass, J. G., \& Corwin, J. (1988). Pragmatics of measuring recognition memory: Applications to dementia and amnesia. Journal of Experimental Psychology: General, 117, 34-50. doi:10.1037/0096-3445.117.1.34

Taatgen, N. A., Juvina, I., Schipper, M., Borst, J. P., \& Martens, S. (2009). Too much control can hurt: A threaded cognition model of the attentional blink. Cognitive Psychology, 59, 1-29. doi:10.1016/j.cogpsych.2008.12.002

Tombu, M., \& Jolicœur, P. (2003). A central capacity sharing model of dual-task performance. Journal of Experimental Psychology: Human Perception and Performance, 29, 3-18. doi:10.1037/ 0096-1523.29.1.3

Visser, T. A. W., Bischof, W. F., \& Di Lollo, V. (1999). Attentional switching in spatial and nonspatial domains: Evidence from the attentional blink. Psychological Bulletin, 125, 458-469. doi:10.1037/0033-2909.125.4.458

Wickens, T. D. (2002). Elementary signal detection theory. New York: Oxford University.

Wilken, P., \& Ma, W. J. (2004). A detection theory account of change detection. Journal of Vision, 4, 1120-1135. doi:10.1167/4.12.11

Woodman, G. F., \& Vogel, E. K. (2005). Fractionating working memory: Memory encoding and maintenance are independent processes. Psychological Science, 16, 106-113. doi:10.1111/ j.0956-7976.2005.00790.x

Wyble, B., Bowman, H., \& Nieuwenstein, M. (2009). The attentional blink provides episodic distinctiveness: Sparing at a cost. Journal of Experimental Psychology: Human Perception and Performance, 35, 787-807. doi:10.1037/a0013902

Zakay, D., \& Block, R. A. (2004). Prospective and retrospective duration judgments: An executive-control perspective. Acta Neurobiologiae Experimentalis, 64, 319-328.

Zakay, D., Tsal, Y., Moses, M., \& Shahar, I. (1994). The role of segmentation in prospective and retrospective time estimation processes. Memory \& Cognition, 22, 344-351. 